\title{
Contents
}

\section{PART ONE Introduction to Spectroscopy}

1 Early Experiments in Spectroscopy $\ldots \ldots \ldots \ldots \ldots \ldots \ldots \ldots \ldots$

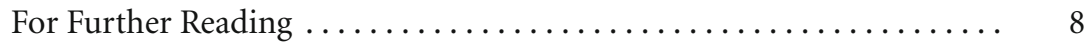

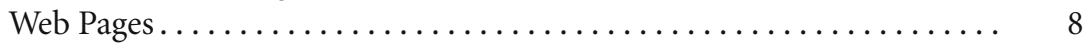

2 A History of Astronomical Spectroscopy ................. 9

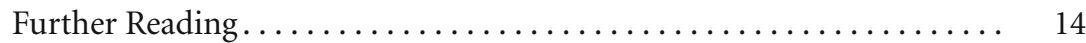

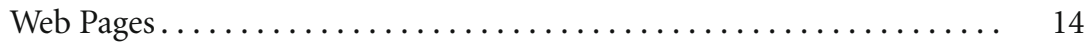

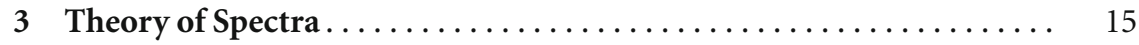

Kirchhoff's Laws ................................... 15

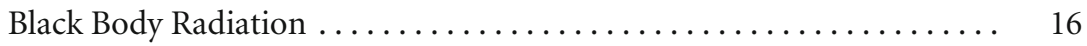

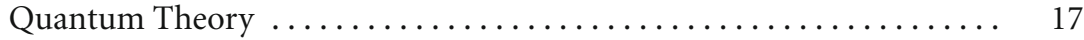

Forbidden Lines................................ 18

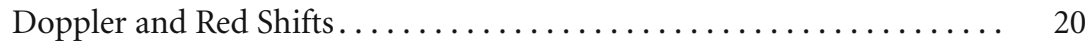

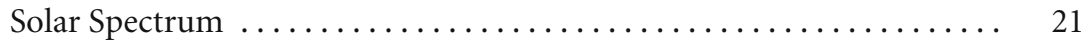

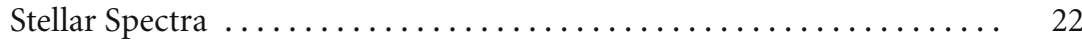

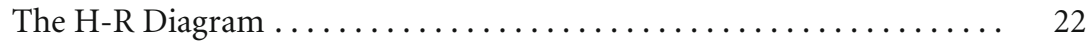

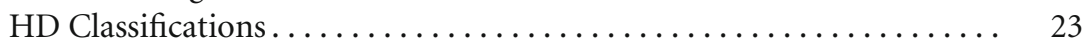

Standard Spectral Lines and Reference Spectra ............... 25

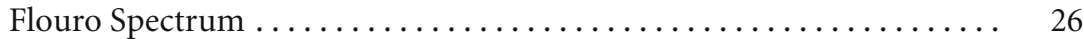

Other Useful Spectral Reference Lines . . . . . . . . . . . . . . 26

Airy Disk, Rayleigh Limits, and FWHM, PSF .............. 28 


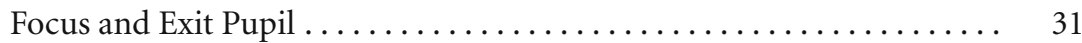

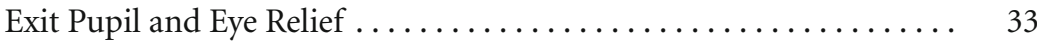

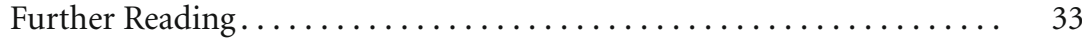

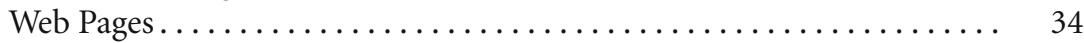

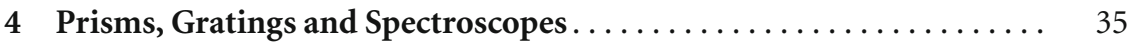

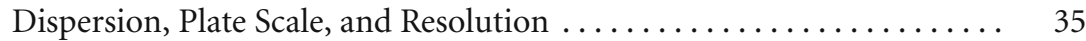

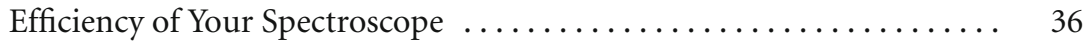

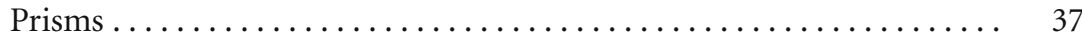

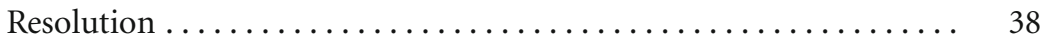

Efficiency of a Prism Spectroscope . . . . . . . . . . . . . . 38

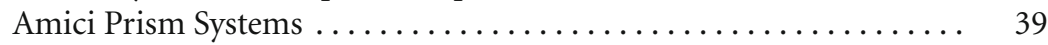

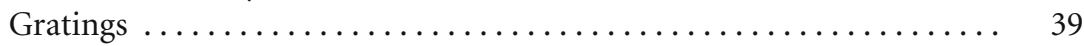

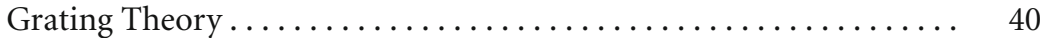

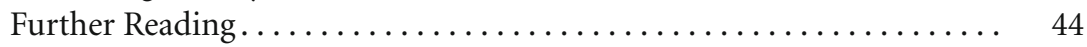

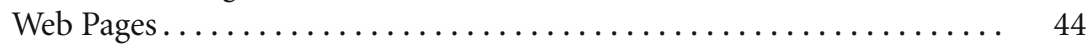

5 Types of Spectroscopes ........................... 45

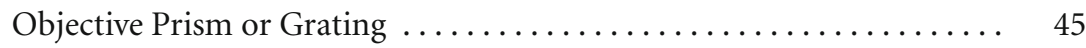

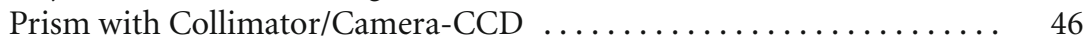

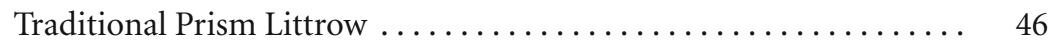

Transmission Grating in Converging Beam .............. 47

Transmission Grating in Collimated Beam (with or without Slit) ... 48

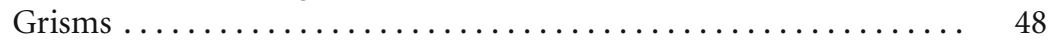

Reflection Grating (with or without Slit) . . . . . . . . . . 49

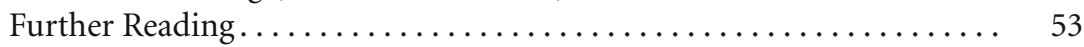

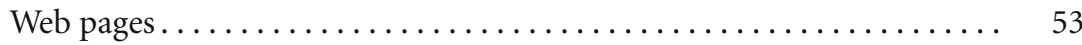

\section{PART TWO Obtaining and Analyzing Spectra}

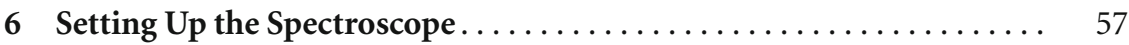

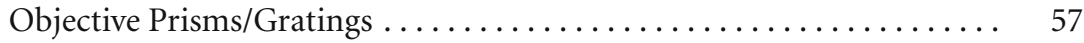

Camera Lenses . . . . . . . . . . . . . . . . . . . . . . . . . . . . . 57

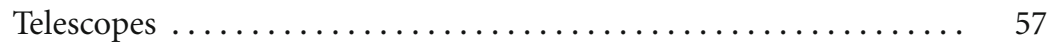

Mounting the Objective Prism/Grating $\ldots \ldots \ldots \ldots \ldots \ldots \ldots \ldots . \ldots \ldots$

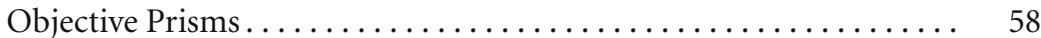

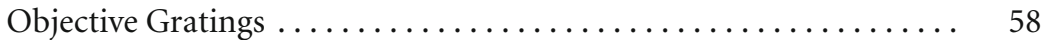

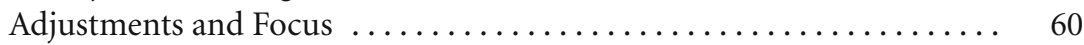

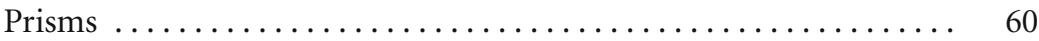

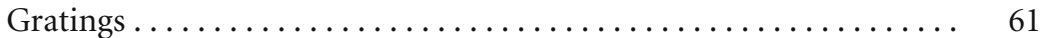

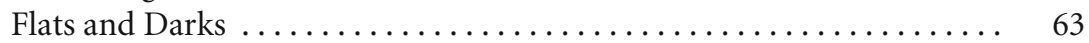

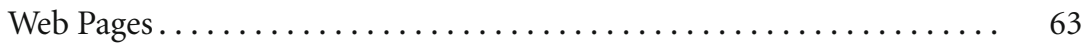

7 Using Spectroscopes in the Converging Beam .............. 65

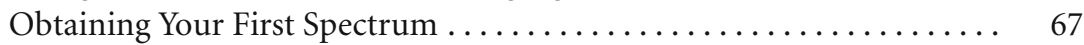

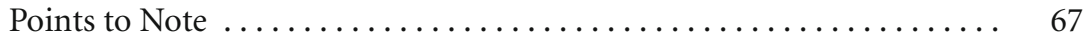


Camera Response vs. Recorded Spectra ................. 68

Using Other Transmission Gratings . . . . . . . . . . . . . . . . 69

Using Gratings in a Collimated Beam $\ldots \ldots \ldots \ldots \ldots \ldots \ldots . \ldots \ldots$

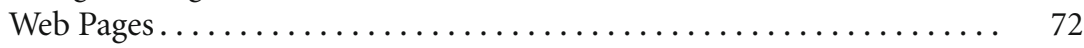

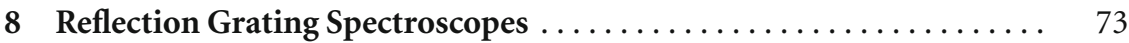

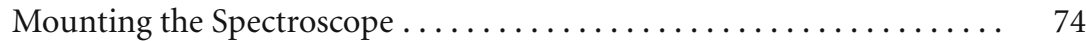

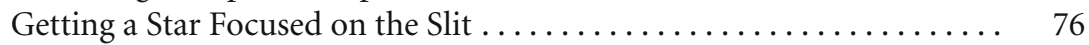

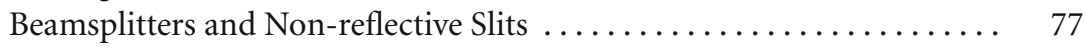

SBIG Software Controlled Spectroscopes................. 77

Baader Dados Spectrograph. . . . . . . . . . . . . . . . . . . . . 79

Shelyak's LhiresIII ............................ 80

Questar QMax Solar Spectrometer .................... 81

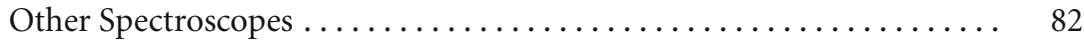

The eShel Spectrograph from Shelyak . . . . . . . . . . . . 82

The Baader Baches Echelle Spectroscope .............. 83

The Optomechanics $10 \mathrm{C} \ldots \ldots \ldots \ldots \ldots \ldots \ldots \ldots \ldots \ldots . \ldots \ldots \ldots$

Remote Control of Spectroscopes .................... 84

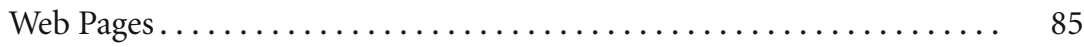

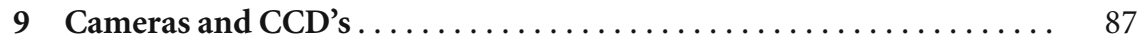

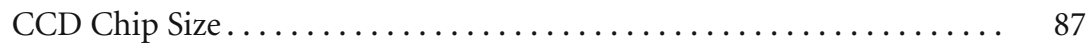

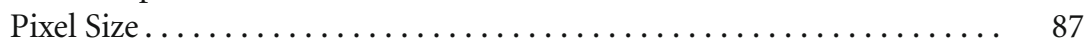

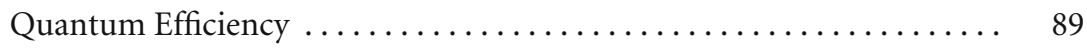

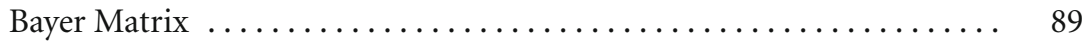

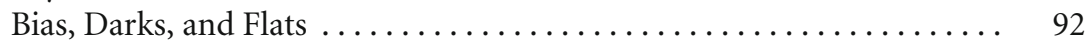

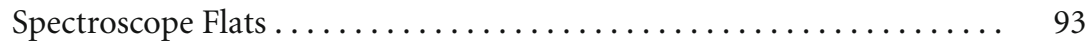

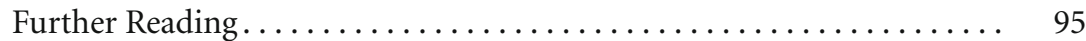

Web Pages................................. 95

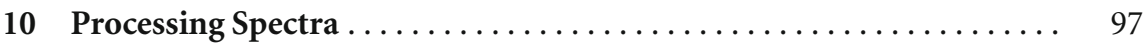

Preparing the Raw Image for Processing $\ldots \ldots \ldots \ldots \ldots \ldots \ldots . \ldots \ldots$

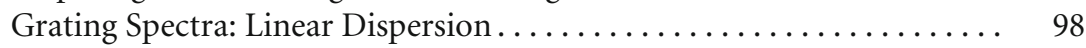

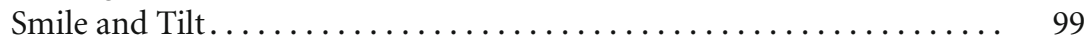

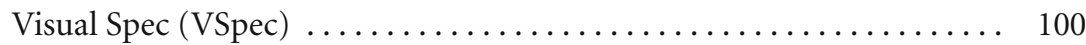

Standard Stellar Spectra . . . . . . . . . . . . . . . . . . . 100

Standard Element Lines . . . . . . . . . . . . . . . . . . . . . 101

Obtaining a Spectral Pixel Profile..................... 101

Wavelength Calibration ......................... 102

Camera Response ............................ 104

Correcting Spectra Using the Camera Response Curve. . . . . . . . . 107

Using the CCD QE Curves - Response Curve . . . . . . . . . . . . 107

Calibrating Using a Reference Lamp . . . . . . . . . . . . . 108

Resolution ................................ 108

Normalized Spectrum $\ldots \ldots \ldots \ldots \ldots \ldots \ldots \ldots \ldots \ldots \ldots \ldots$

Signal to Noise Ratio $(\mathrm{SNR}) \ldots \ldots \ldots \ldots \ldots \ldots \ldots \ldots \ldots \ldots \ldots$ 
Continuum Removal ............................ 109

Equivalent Width $(\mathrm{LEQ}) \ldots \ldots \ldots \ldots \ldots \ldots \ldots \ldots$

Web Pages...................................... 109

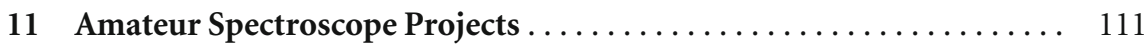

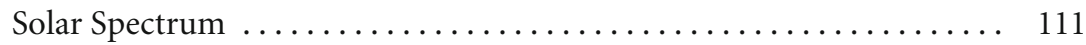

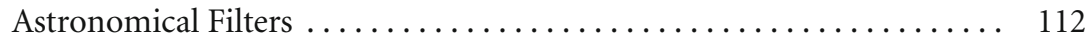

Stellar Classification ............................... 114

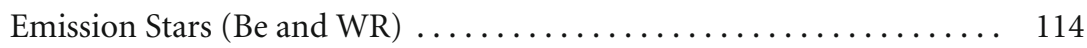

Doppler Shift - Binary Stars/Exoplanets/Quasars . . . . . . . . . . . 117

Variable Stars, Nova, and Supernovae . . . . . . . . . . . . . . . . . 119

Nebulae..................................... 121

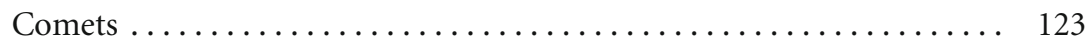

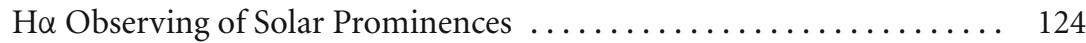

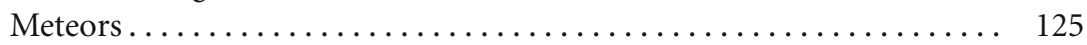

Planetary Spectroscopy. . . . . . . . . . . . . . . . . . 127

Further Reading. ................................ 129

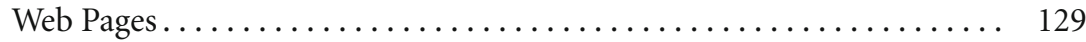

\section{PART THREE Spectroscope Design and Construction}

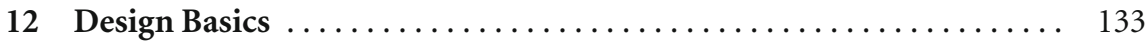

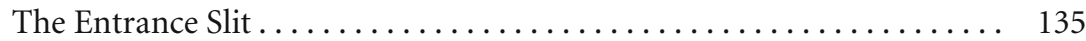

Classic Semi-fixed Entrance Slit . . . . . . . . . . . . . . . . 138

Adjustable Entrance Slits . . . . . . . . . . . . . . . . . . . 139

Commercial Slits ........................... 139

Setting the Slit Gap ......................... 140

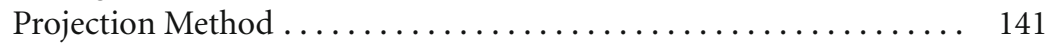

Other Slit Alternatives . . . . . . . . . . . . . . . . . . . . . . 142

Photographic Negative for Slit . . . . . . . . . . . . . . . . . . 142

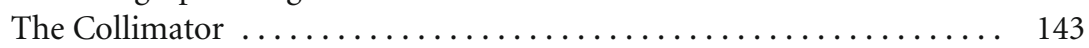

The Prism as a Dispersion Element ..................... 144

Resolution ......................................... 147

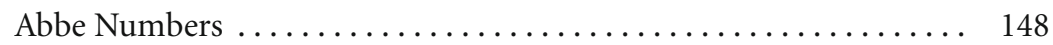

Gratings as a Dispersion Element .................. 148

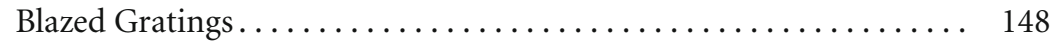

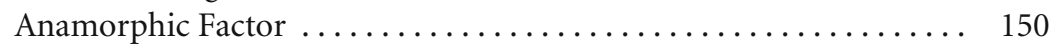

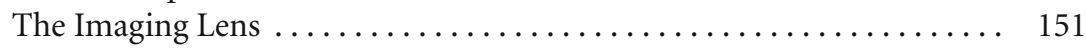

The Eyepiece/Camera/CCD . . . . . . . . . . . . . . . . . . . 152

Attaching and Focusing the Camera/CCD . . . . . . . . . . . . 153

Spectroscope Design Summary . . . . . . . . . . . . . . . 153

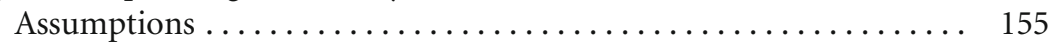

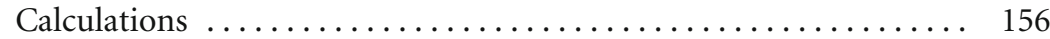

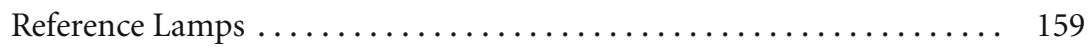

Testing and Calibrating Spectroscopes $\ldots \ldots \ldots \ldots \ldots \ldots \ldots \ldots \ldots$ 
Laser Collimator . . . . . . . . . . . . . . . . . . . . . . . . . 161

Testing the Collimator Alignment and Focus . . . . . . . . . . 162

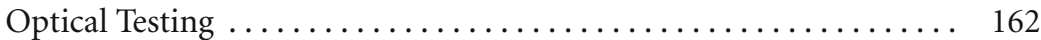

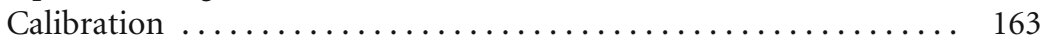

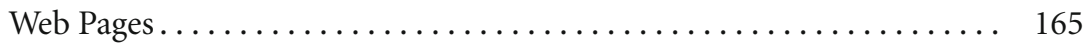

13 Prism Spectroscope Designs $\ldots \ldots \ldots \ldots \ldots \ldots \ldots \ldots \ldots \ldots \ldots \ldots \ldots \ldots$

Objective Prisms ............................... 167

Other Prism Spectroscopes ........................ 169

Prisms in Collimated Beams . . . . . . . . . . . . . . . . . . . . 169

Traditional Prism Spectroscope . . . . . . . . . . . . . . . . . 171

Classic Littrow Prism Spectroscope . . . . . . . . . . . . . . . 172

Efficiency of a Prism Spectroscope . . . . . . . . . . . . . . 173

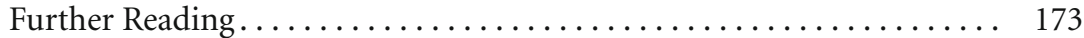

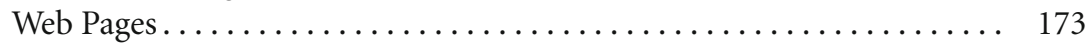

14 Transmission Grating Spectroscope Designs ................ 175

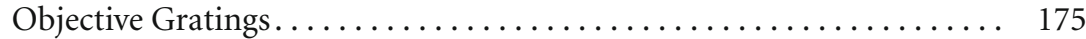

Converging Beam Arrangement . . . . . . . . . . . . . . . 175

Aperture Mask ......................... 181

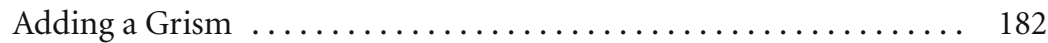

Adding a Slit . . . . . . . . . . . . . . . . . . . . . . . . 184

Mounting Other Transmission Gratings in a Converging Beam . . . . 186

Improving Resolution with a Collimator Lens . . . . . . . . . . 188

Using a Barlow Lens as a Collimator . . . . . . . . . . . . . . . . 189

Watkis Transmission Grating Spectroscope ................... 189

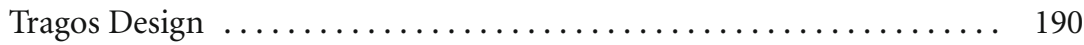

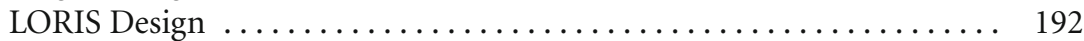

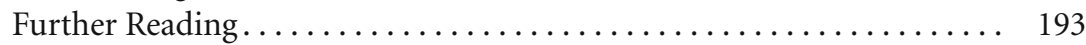

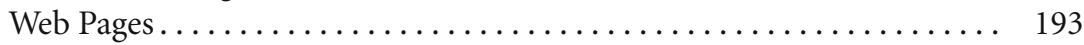

15 Reflection Grating Spectroscope Designs . . . . . . . . . . . . . . . 195

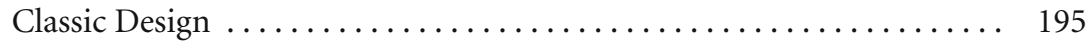

Construction Notes ........................ 196

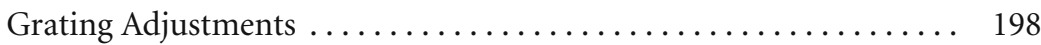

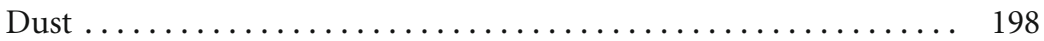

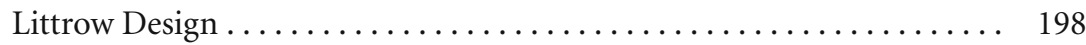

Construction Notes ............................. 199

Grating Housing and Adjustment . . . . . . . . . . . . . . 200

Camera/Focuser ................................ 201

Reference Light Source ... . . . . . . . . . . . . . . . . . . 202

Slit Back Light Arrangement . . . . . . . . . . . . . . . . . . 203

WPO Design ................................ 203

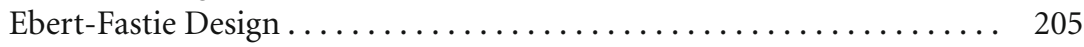

Czerny-Turner Design ........................... 209

Echelle Spectroscopes ............................... 209 


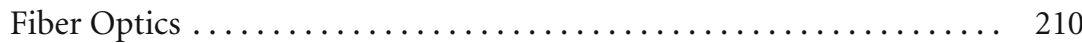

Aperture Ratio, F/Ratio, and Numerical Aperture (NA) . . . . . . 211

Fiber Optic Applications . . . . . . . . . . . . . . . . . . . . 211

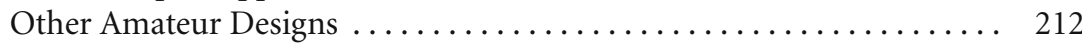

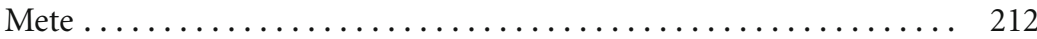

Kaye ................................... 214

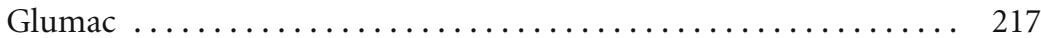

Bareges CCD Spectrograph (Rondi/Buil) ............... 217

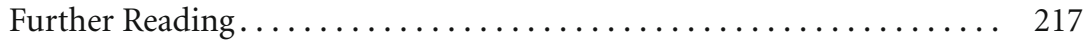

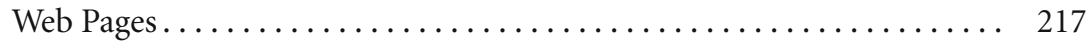

16 Guiding, OAG, Beamsplitters, and Flip Mirrors . . . . . . . . . . . 219

Off-Axis Guiders ............................... 219

Beamsplitter Spectroscope Applications .................... 219

Modifying a Vixen Flip Mirror to a Beamsplitter . . . . . . . . . 220

Fitting and Adjusting the Beamsplitter Plate ............. 223

Using the Beamsplitter............................ 224

Dove Prism as Beamsplitter ....................... 224

Transfer Systems for Guide Cameras . . . . . . . . . . . . . 226

Split Mirror Guider ........................... 226

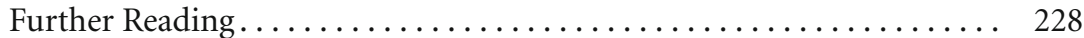

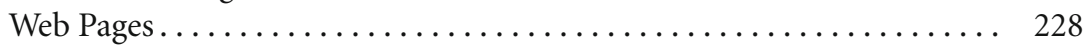

Appendix A ................................... 229

Suppliers of Spectroscopes and Accessories . . . . . . . . . . . . . 229

Star Analyser Grating . . . . . . . . . . . . . . . . . . . . 229

Rainbow Optics Star Spectroscope . . . . . . . . . . . . . . . . . . . 229

SGS and DSS-7 Spectroscopes .................... 229

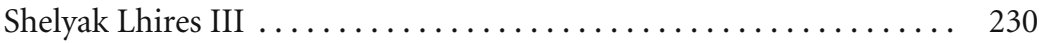

Baader Dados Spectrograph ...................... 230

Rigel Systems................................. 230

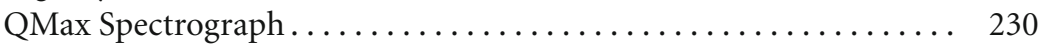

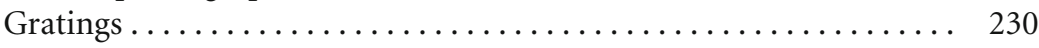

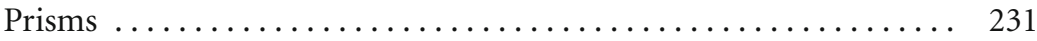

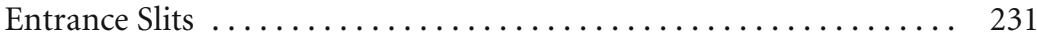

Lenses and Mirrors........................ 231

Electroluminescent Panels........................ 231

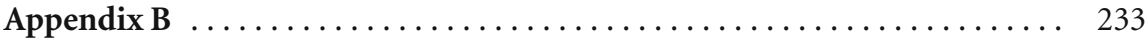

Useful Spectroscopy Forums and Other Websites............ 233

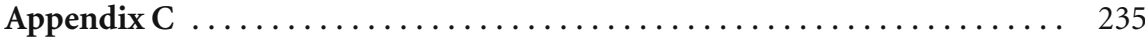

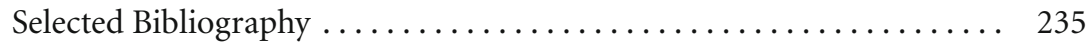

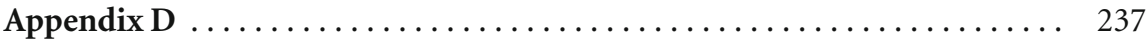

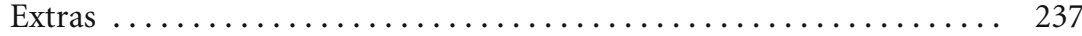

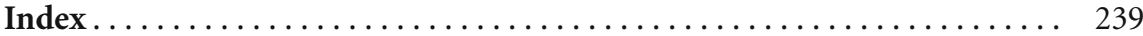

